\title{
PABRE-Man: Management of a Requirement Patterns Catalogue ${ }^{\S}$
}

\author{
Cristina Palomares, Carme Quer, Xavier Franch \\ Software Engineering for Information Systems research group (GESSI) \\ Universitat Politècnica de Catalunya (UPC) \\ Barcelona (Catalunya, Spain) \\ \{cpalomares, cquer, franch\}@essi.upc.edu
}

\begin{abstract}
Software requirement patterns have been proposed as an artifact for fostering requirements reuse. In this paper we present PABRE-Man, a software subsystem aimed at managing a catalogue of patterns ready to be applied in requirements engineering projects.
\end{abstract}

Keywords-software requirement patterns, requirements reuse, call-for-tender processes.

\section{INTRODUCTION}

The reuse of software requirements may help requirement engineers to elicit, validate and document software requirements and as a consequence, obtain Software Requirement Specifications (SRS) of better quality both in contents and syntax. There are many approaches to reuse. Among them, patterns hold a prominent position. A Software Requirement Pattern (SRP), following the typical contextproblem-solution scenario of patterns, basically consists of: a template that may generate one or more requirements by tailoring the template (solution) to a certain project, and some information (context-problem) to identify its need to be applied in that particular project.

In this paper we present PABRE-Man, which is one of the tools that compose the PABRE system (Fig.1): PABREMan facilitates the definition of SRPs, as well as the maintenance and evolution of an SRP catalogue; PABREProj provides the use of the SRP catalogue during the elicitation stage and the generation of SRS or call-for-tenders documents.

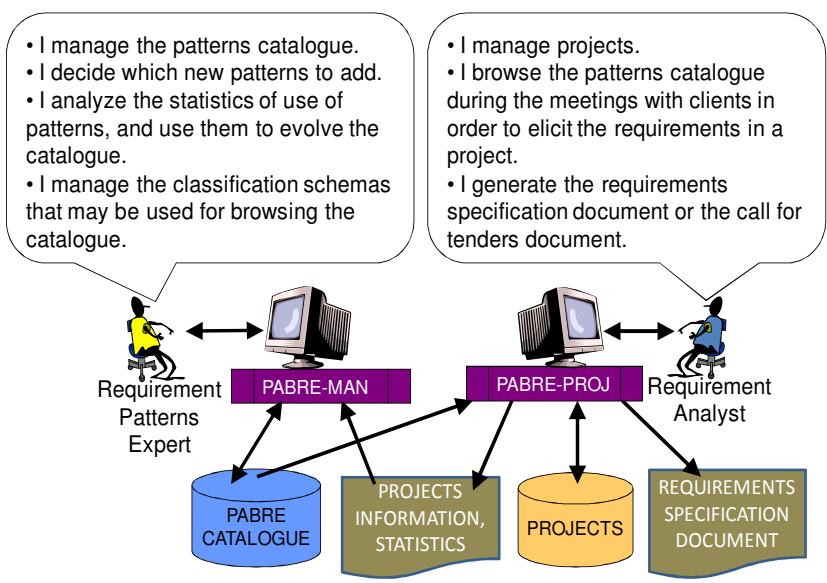

Figure 1. The PABRE system

\footnotetext{
${ }^{\S}$ This work has been supported by the project TIN2010-19130-C02-01.
}

Using SRPs in a certain software project implies applying them to elicit the requirements of the project. In the case of requirements that are not covered by any SRP, it is possible to create new requirements related to an SRP (but not as direct application) or even with no relationship to any SRP. The new requirements of different projects can be considered during the catalogue evolution to generate new SRPs or change existent ones.

Both tools have been developed to be the technological support to the assets that we are obtaining in our collaboration with the SSI group of the Centre de Reserche Publique Henri Tudor (CRPHT) of Luxembourg. Specifically, these assets are: one SRP catalogue [1], that is currently composed of 29 non-functional SRPs, and that is continuously evolving; and the PABRE method [2], that provides a guide of use of the SRP catalogue.

\section{SOFTWARE REQUIREMENT PATTERNS}

For space reasons, we do not include in this paper the metamodel that describes SRPs [3], and on which has been based the development of the tools. Thus, in order to facilitate the understanding of the tool we present SRPs by means of an example. An SRP is a pattern that, when applied, produces requirements related to the objective (goal) of that pattern. For instance, the SRP Failure Alerts (Fig.2) application would produce requirements related to the goal of having a system that alerts users about system failures (which types of failures shall be monitored, what types of alerts shall be launched, etc.). Goals correspond to problems to be solved by applying the SRP.

An SRP consists of several Forms, which represent different solutions for achieving the goal. For example, the Failure Alerts pattern has two different Forms depending on whether there is the need of having specific types of alerts depending on the types of failures or not. In each Form we always find a Fixed Part and some Extended Parts (both being templates). The Fixed Part always becomes a requirement when using the Form. Extended Parts are only used if more precise information is required. Fixed and Extended Parts are both composed by the text to be used as a requirement and optionally some parameters (in italics in the example) to be instantiated during the pattern application in a given software project. The domain of possible values of these parameters is constrained by stating their metrics, which in our example correspond to the set of possible alert types and the set of possible failure types. 


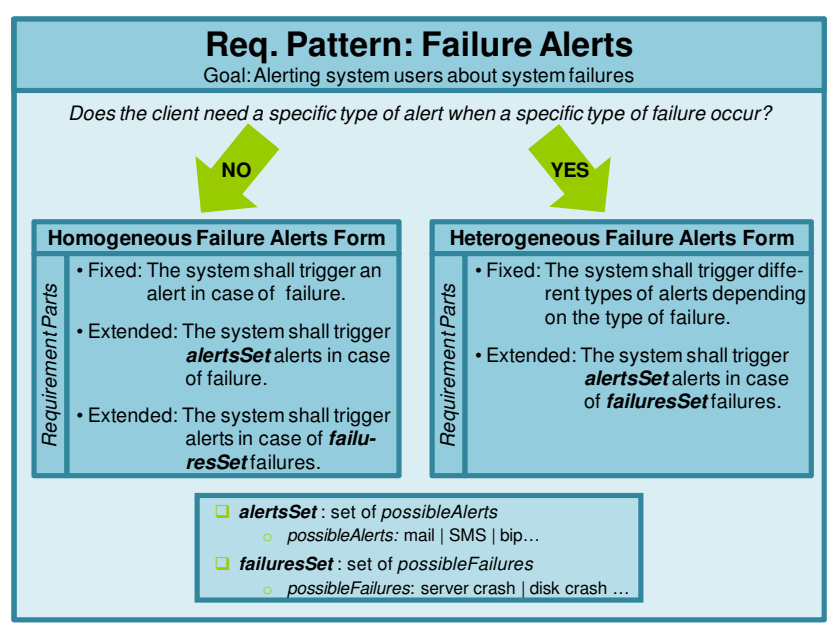

Figure 2. The Failure Alerts SRP

The Schemas are hierarchies of classifiers that facilitate the identification and organization of the SRPs relevant for one project. For usability purposes, each requirement analyst may define his/her own schemas. These classification schemas give the context where each SRP make sense.

\section{PABRE-MAN DESCRIPTION}

The functionalities provided by PABRE-Man are mainly: edition and classification of SRPs; exportation of the catalogue (to pdf and rtf); importation of data of the SRPs use in software projects. This importation is relevant for catalogue evolution, and takes to the tool information about: 1) how many times an SRP has been applied; 2) new requirements related with an SRP but not as a direct SRP application; 3) new requirements not related to any existent SRP.
The interface of the tool (Fig. 3) shows permanently a tree with the SRPs of the catalogue classified taking into account one schema. For example, in the figure the catalogue is shown classified following the characteristics and subcharacteristics of the ISO 9126-1 standard. The screenshot corresponds to the edition of SRP Failure Alerts. Specifically, it is possible to see one Extended Part of this SRP with its text and the parameters alerts and failures associated to it, and the type of the metrics of each one. In the main window the metrics manager presents metrics already defined and available for the parameters definition.

\section{FINAL REMARKS}

More details about how the current SRP catalogue has been constructed, the PABRE method and the SRP metamodel can be found in $[1,2,3]$.

Ddifferent scenarios of use are presented in [1]. Currently both tools are beginning to be used in certain software procurement projects conducted by the CRPHT, by means of the integration of PABRE tools with OPAL [4], the requirements management tool (RMT) used by the CRPHT.

As future work, a new release of PABRE-Man is being developed that provides having versions of SRPs, and exporting requirements to several marketplace RMTs.

\section{REFERENCES}

[1] http://www.upc.edu/gessi/PABRE/

[2] S. Renault, O. Mendez-Bonilla, X. Franch, C. Quer: A pattern-based method for building requirements documents in call-for-tender processes. IJCSA 6(5): 175-202 (2009)

[3] X. Franch, C. Palomares, C. Quer, S. Renault, F. De Lazzer: A Metamodel for Software Requirement Patterns. REFSQ'10.

[4] M. Krystkowiak, V. Bétry, E. Dubois. Efficient COTS Selection with OPAL Tool. MPEC 2004.

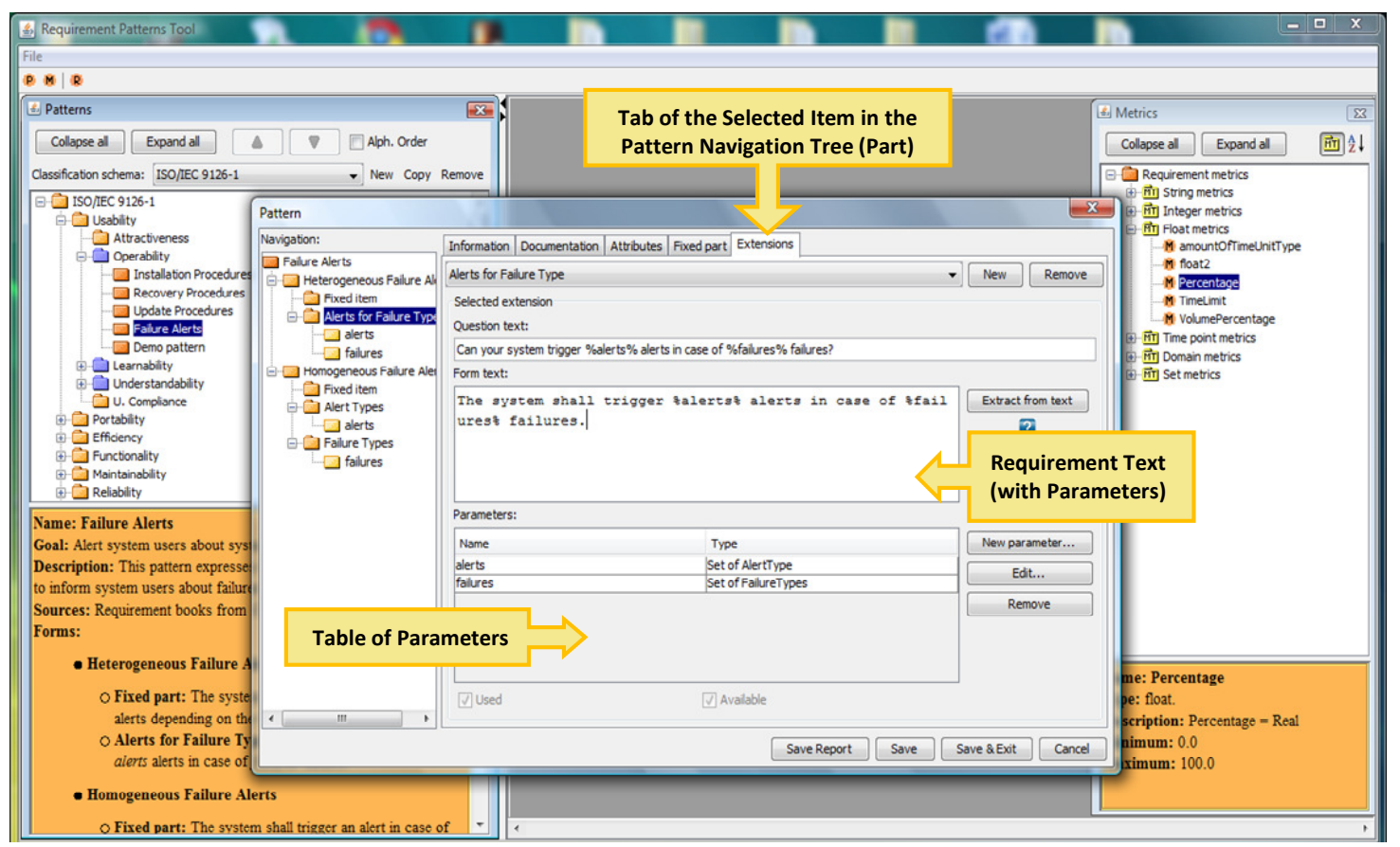

Figure 3. PABRE-Man screenshot 